\title{
Discovery of Sodium-Doped Triphenylene Superconductors by Searching the Organic Material Database
}

Taekyung Yoon ${ }^{\dagger, \ddagger}$, Ina Park ${ }^{\dagger, \ddagger}$, Thao Phuong Nguyen ${ }^{\dagger}$, Duck Young Kim ${ }^{\uparrow}$, , Jinho Lee ${ }^{\dagger}$, Ji Hoon Shim ${ }^{*}, \uparrow$, , Hee Cheul Choi ${ }^{*, \dagger}$

${ }^{\dagger}$ Department of Chemistry, Pohang University of Science and Technology (POSTECH), Pohang 37673, Republic of Korea

$\S$ Department of Physics, Pohang University of Science and Technology (POSTECH), Pohang 37673, Republic of Korea

^ Center for High Pressure Science and Technology Advanced Research (HPSTAR), Shanghai 201203, China

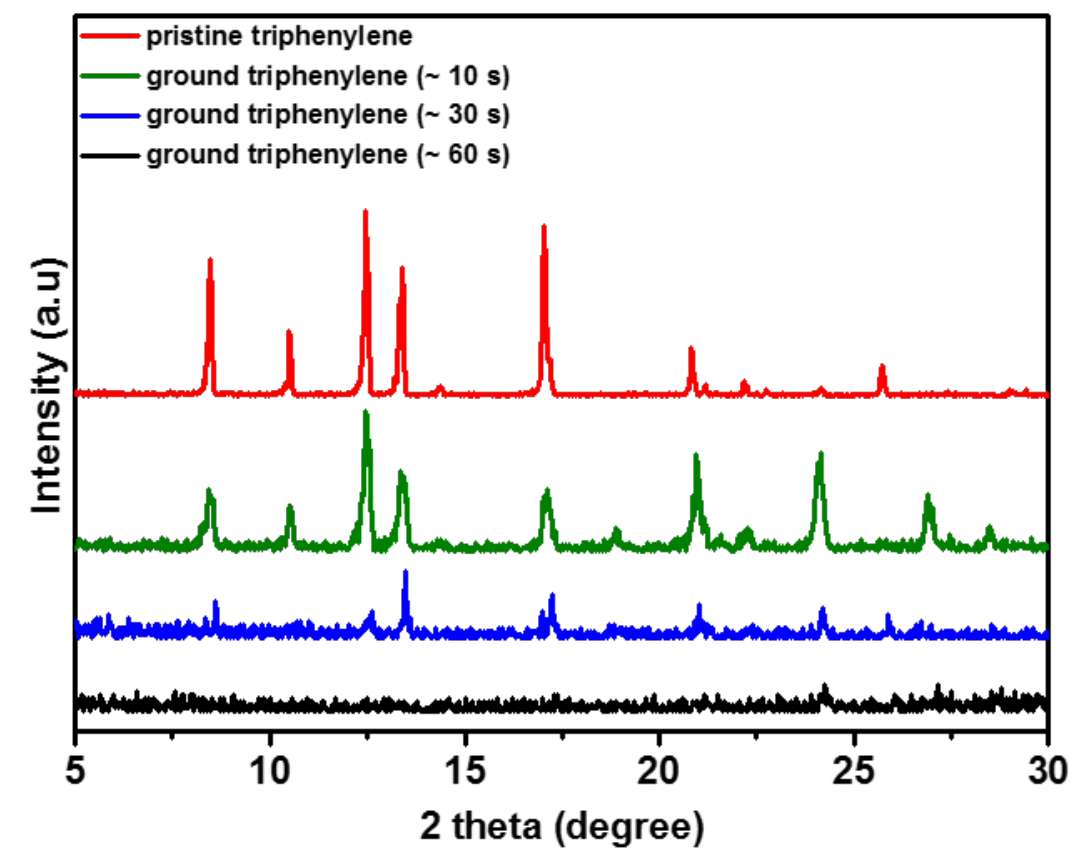

Figure S1. X-ray diffraction patterns for pristine and directly ground triphenylene with sodium samples depending on grinding time. Pristine triphenylene powder (red), for 10, 30 seconds grinding with sodium (green, blue) and for 1 minute grinding with sodium (black). 

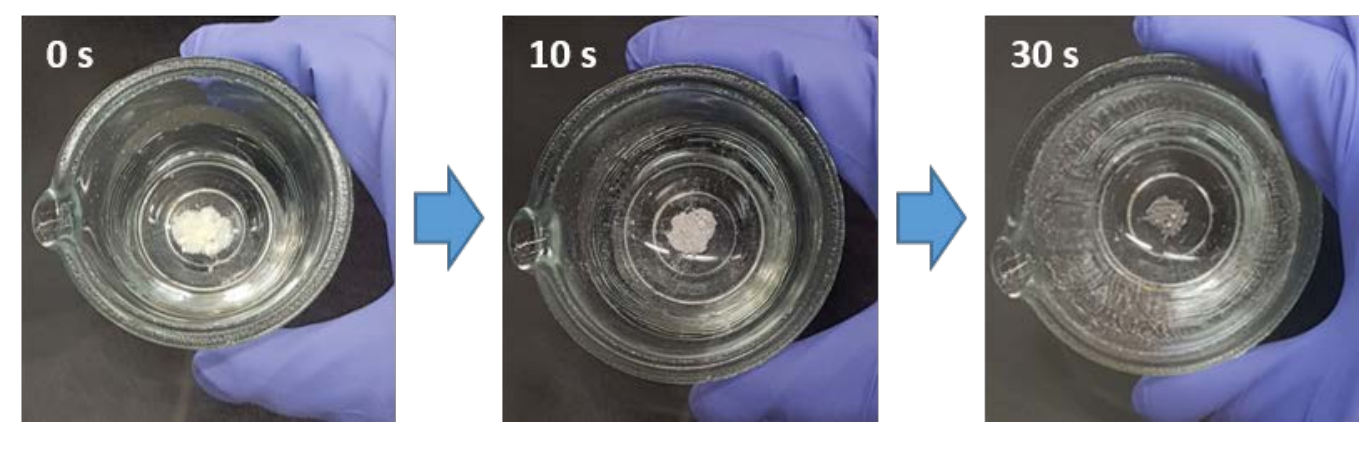

Figure S2. Real images of color changing in triphenylene during grinding with sodium depending on time. 


\section{Search for superconducting candidate among PAH database}

The list of 200 polycyclic aromatic hydrocarbon (PAH) molecules were obtained from the Cambridge Structural Database (CSD) and PubChem - 55 molecules from CSD and 145 molecules from PubChem. The distribution of $200 \mathrm{PAH}$ molecules are shown in figure 1 and it shows that they have various molecular sizes. It is not shown here, but they also have various kind of geometrical shapes.

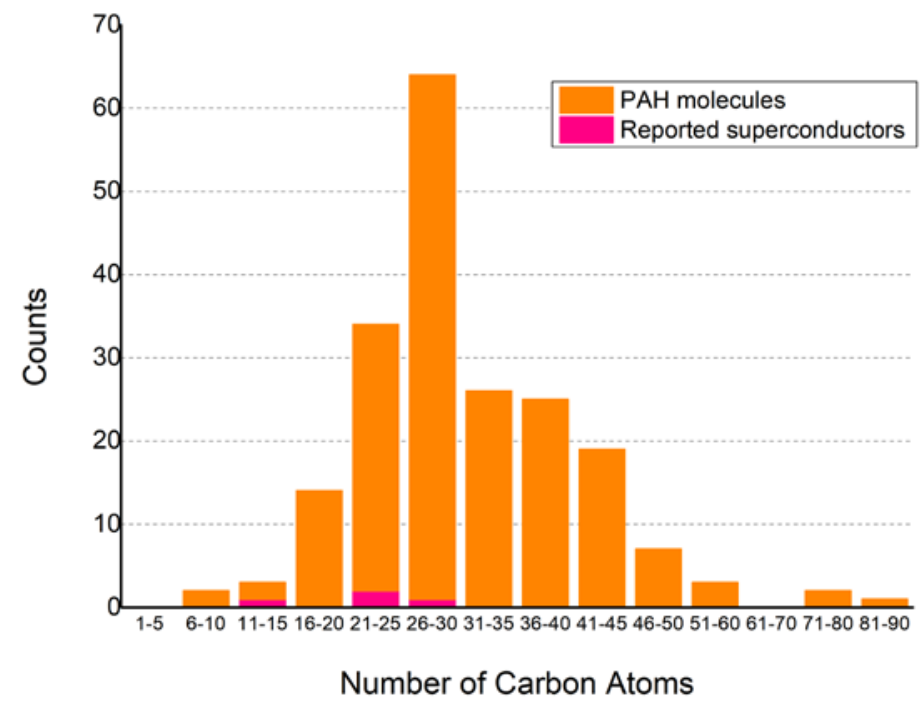

Figure S3. Distribution of 200 PAH molecule data from CSD and PubChem.

The structure of 200 PAH molecules were relaxed first in DFT with B3LYP exchange-correlation functional and 6-31G(d,p) basis. Then, $\Delta$, the energy difference of LUMO and LUMO+1 states of each molecules were obtained. The distribution of $\Delta$ was random regardless of the size or the geometrical shape of molecules. We could select 34 molecules with very small $\Delta$ under $0.2 \mathrm{eV}$. (Table 1 ) The value of 0.2 for $\Delta$ is the smallest value that can contain PAH superconductors reported so far. Note that, for 1,2:8,9-dibenzopentacene, it has very large $\Delta$ value of $1.2 \mathrm{eV}$, however, it has small gap between LUMO+1 and LUMO+2 states of about $0.12 \mathrm{eV}$. Similarly, p-terphenyl, also proposed as possible aromatic hydrocarbon superconductor, has large $\Delta$ value of $0.89 \mathrm{eV}$ but a small gap size of $0.11 \mathrm{eV}$ between LUMO+1 and LUMO+2 states.

Among 34 PAH molecules with small $\Delta$, we have searched for some molecules with experimentally known stacking type. (Table 1) Not many stacking type of molecules were known, but here, we could find 6 molecules with herringbone type and 3 molecules with $\gamma$-motif stacking. Since picene and phenanthrene have herringbone stacing type and coronene have $\gamma$-motif stacking, we newly found 4 and 2 molecules with each type of stacking have high possibility to show superconductivity, respectively. Among them, triphenylene have the smallest value of $\Delta$. 
Table S1. List of 34 selected PAH molecules with $\Delta$ under $0.2 \mathrm{eV}$.

\begin{tabular}{|c|c|c|c|c|}
\hline No. & Name & Molecular Structure & $\Delta(\mathrm{eV})$ & Stacking \\
\hline 1 & $\begin{array}{c}\text { 10,10'-Diphenyl- } \\
\text { 9,9'-bianthryl }\end{array}$ & & 0.0000 & $\beta-\mathrm{HB}^{1)}$ \\
\hline 2 & $\begin{array}{c}\text { Hexabenzo[bc,ef, } \\
\text { hi,kl,no,qr] } \\
\text { coronene }\end{array}$ & & 0.0000 & Y-motif2) \\
\hline 3 & $\begin{array}{c}\text { Tribenzo }(a, d, g) c o \\
\text { ronene }\end{array}$ & & 0.0000 & - \\
\hline 4 & $\begin{array}{c}\text { Hexabenzo[a,d,g,j } \\
\text {,m,p]coronene }\end{array}$ & & 0.0000 & - \\
\hline 5 & $\begin{array}{l}\text { Hexacyclopentac } \\
\text { oronene }\end{array}$ & & 0.0000 & - \\
\hline 6 & Coronene & & 0.0000 & Y-motif3) \\
\hline 7 & Benzene & & 0.00027 & Herringbone ${ }^{4)}$ \\
\hline 8 & Kekulene & & 0.00027 & Y-motif5) \\
\hline 9 & Triphenylene & & 0.00054 & Herringbone ${ }^{1)}$ \\
\hline 10 & $\begin{array}{l}\text { Acenaphtho[3,21, } \\
\text { 8- } \\
\text { fghij]tetrabenzo[a, } \\
\text { c,m,o]picene }\end{array}$ & & 0.00082 & $\beta$-motif1) \\
\hline
\end{tabular}




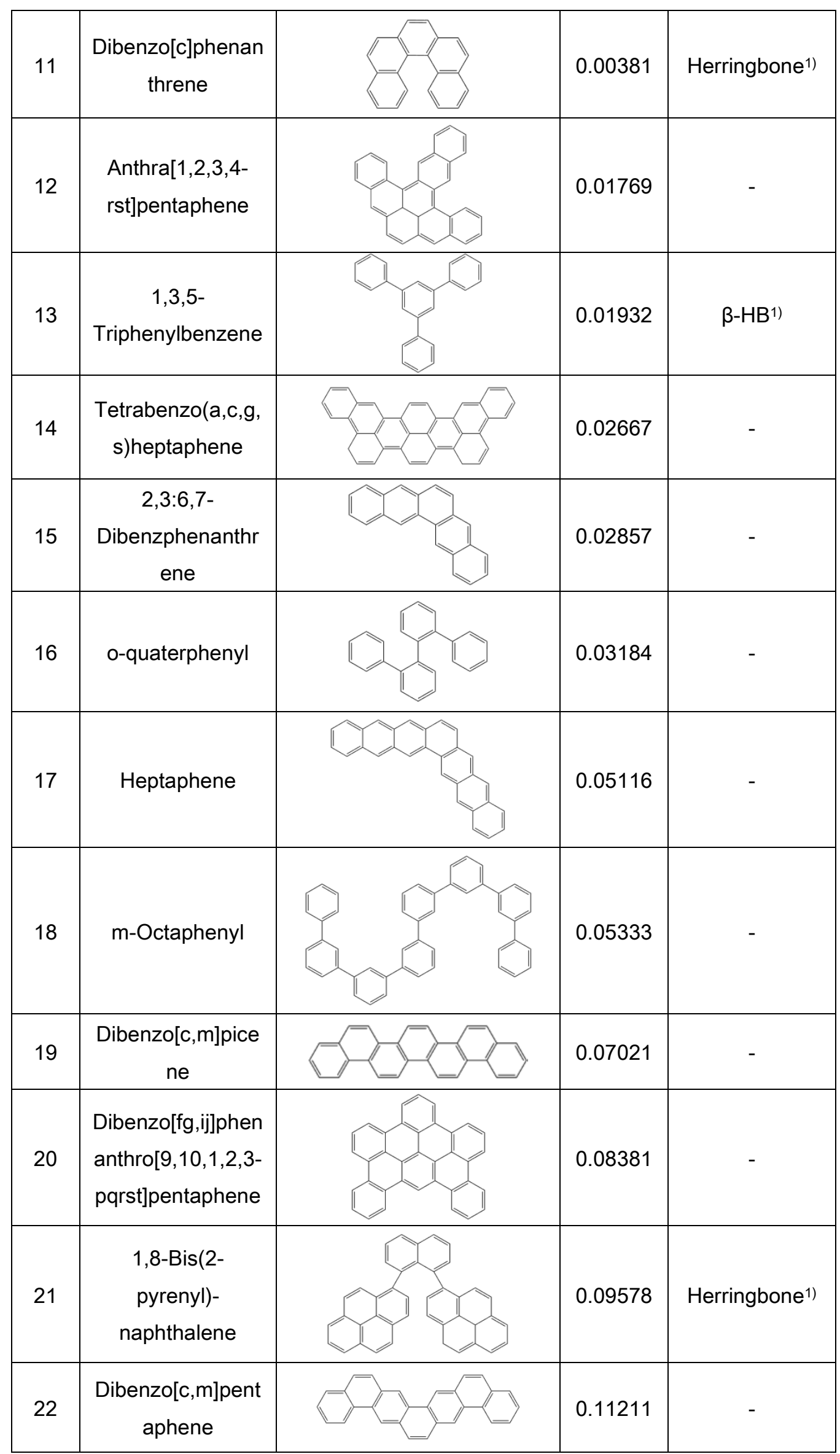




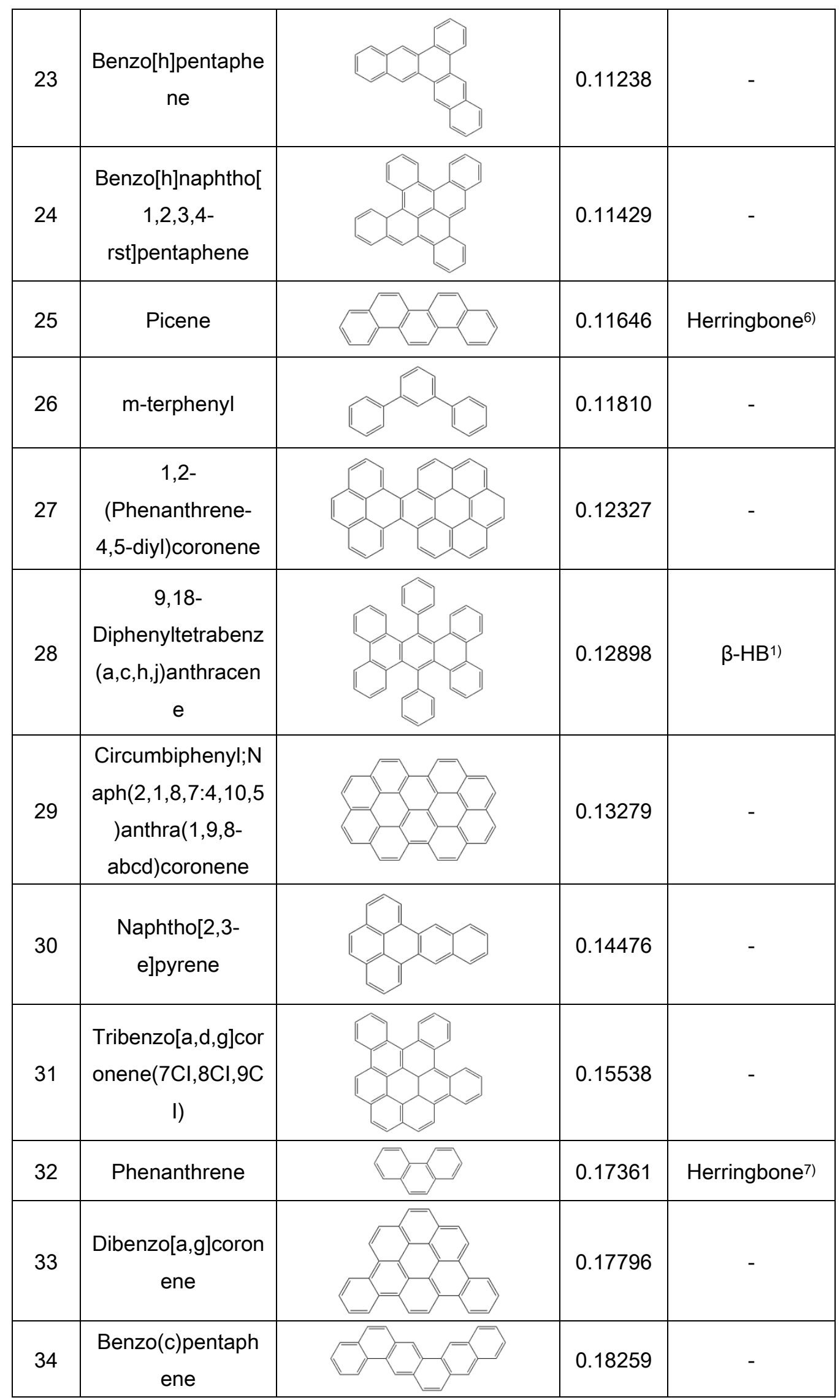


a

\begin{tabular}{|c|c|c|c|c|}
\hline Entry \# & $\begin{array}{c}\text { Sample } \\
\text { (nominal name) }\end{array}$ & $\begin{array}{c}\text { Annealing } \\
\text { temperature }\left({ }^{\circ} \mathrm{C}\right)\end{array}$ & $\begin{array}{l}\text { Reaction } \\
\text { time (h) }\end{array}$ & $\begin{array}{l}\text { Glovebox condition } \\
\text { (moisture / oxygen) }\end{array}$ \\
\hline 1 & \multirow{10}{*}{$\mathrm{Na}_{3}$ triphenylene } & \multirow{10}{*}{198} & 20 & 2.0 p.p.m. / 0.3 p.p.m. \\
\hline 2 & & & 20 & 2.6 p.p.m. / 0.1 p.p.m. \\
\hline 3 & & & 20 & 1.7 p.p.m. / 0.1 p.p.m. \\
\hline 4 & & & 20 & 3.1 p.p.m. / 0.2 p.p.m. \\
\hline 5 & & & 20.5 & 2.5 p.p.m. / 0.4 p.p.m. \\
\hline 6 & & & 20 & 2.0 p.p.m. / 0.2 p.p.m. \\
\hline 7 & & & 20 & 1.6 p.p.m. / 0.1 p.p.m. \\
\hline 8 & & & 20 & 2.6 p.p.m. / 0.3 p.p.m. \\
\hline 9 & & & 20 & 2.0 p.p.m. / 0.1 p.p.m. \\
\hline 10 & & & 20 & 1.6 p.p.m. / 0.1 p.p.m. \\
\hline
\end{tabular}
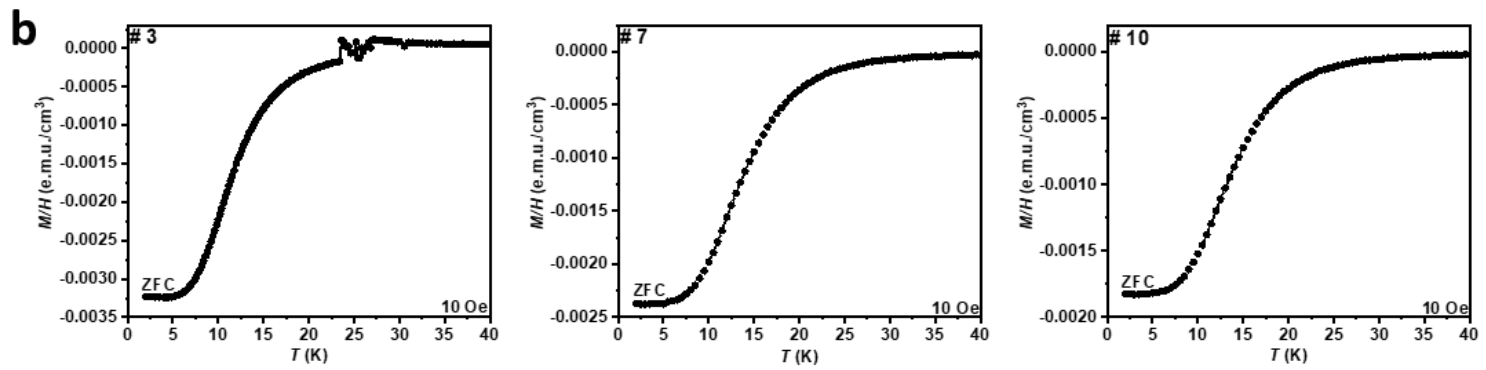

Figure S4. Information on 10 consecutive samples measured for reproducibility of sodium-doped triphenylene superconductor. (a) Table for data of the samples used for the reproducibility test. (b) $M / H$ versus $T$ plots for the measurement of ZFC from superconducting sodium-doped triphenylene (from entry number of 3,7 , and 10 ). 

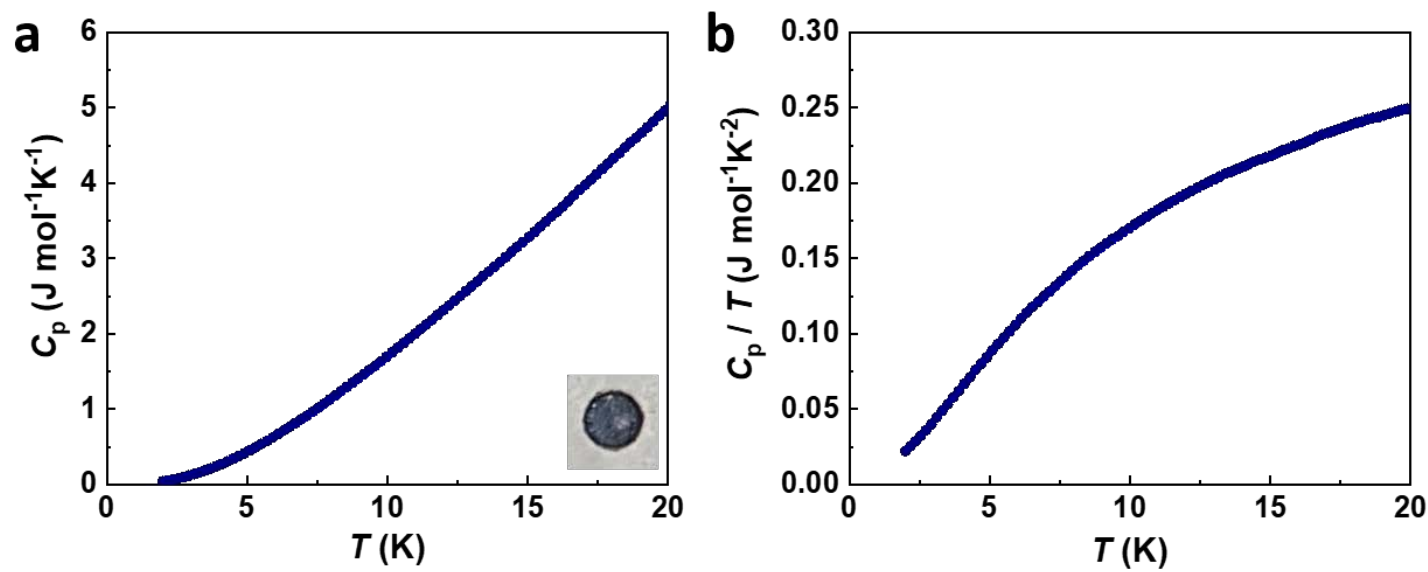

Figure S5. (a) Heat capacity and (b) specific heat of copper powder contained sodium-doped triphenylene (CuTPH) pellet ( $2-20 \mathrm{~K})$. (Inset) CuTPH pellet used for measurement. 


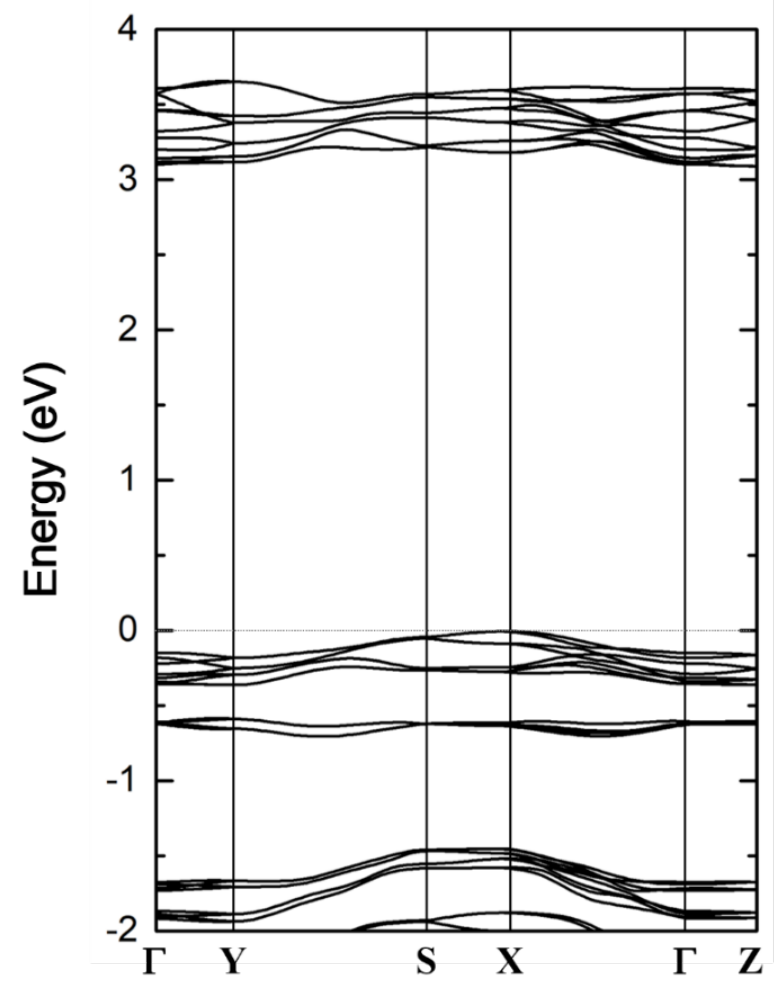

Figure S6. Band structure of pristine triphenylene crystal. 

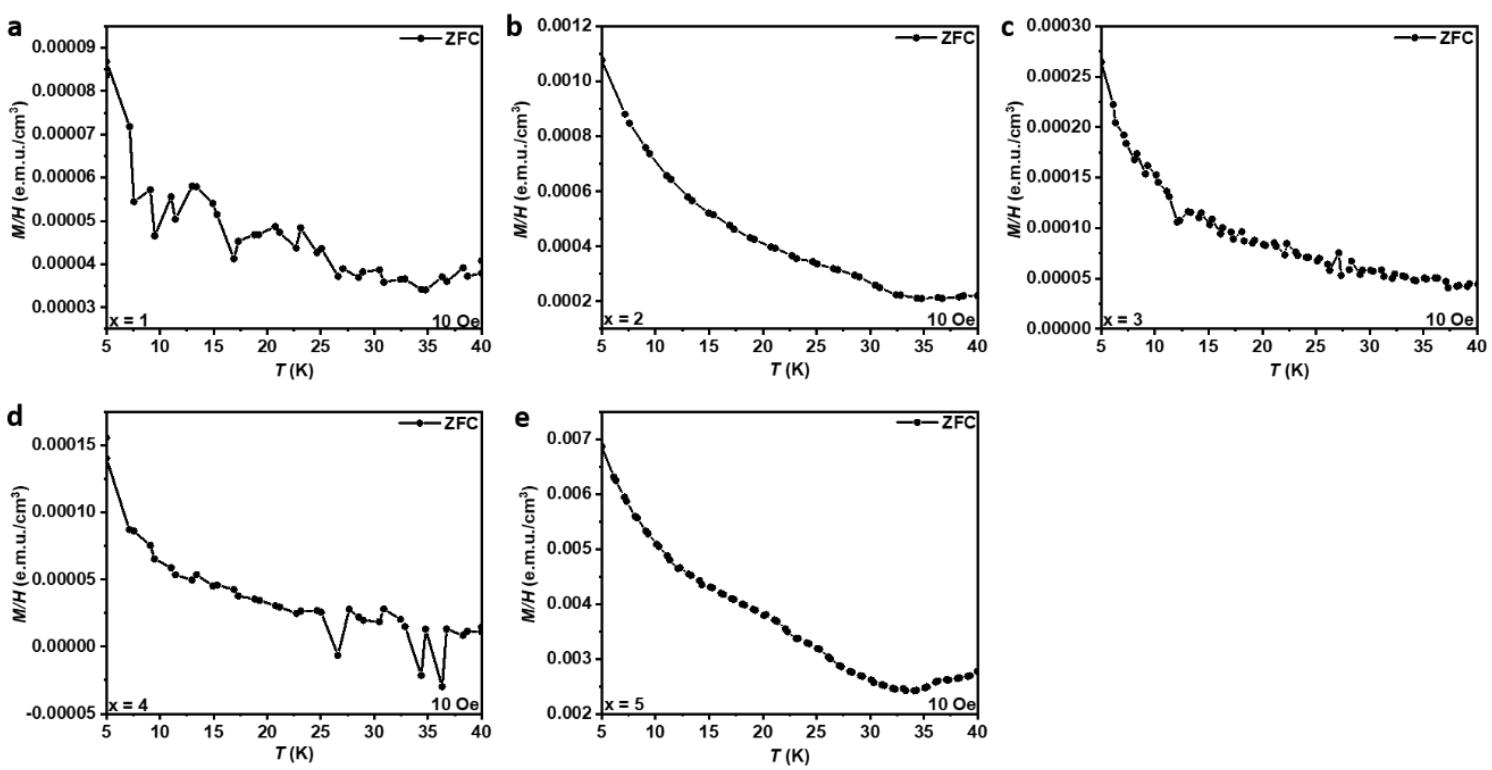

Figure S7. Magnetization of $\mathrm{K}_{\mathrm{x}}$ triphenylene. $\mathrm{K}_{\mathrm{x}}$ triphenylene prepared with grinding-annealing method. All sample shows paramagnetic properties. (a) $x=1$, (b) $x=2$, (c) $x=3$, (d) $x=4$, (e) $x=5$, respectively. 


\section{References}

1) Schatschneider, B.; Phelps, J.; Jezowski, S. A new parameter for classification of polycyclic aromatic hydrocarbon crystalline motifs: a Hirshfeld surface investigation. CrystEngComm 2011, 13, 7216-7223.

2) Kübel, C.; Eckhardt, K.; Enkelmann, V.; Wegner, G.; Müllen, K. Synthesis and crystal packing of large polycyclic aromatic hydrocarbons: hexabenzo[bc,ef,hi,kl,no,qr]coronene and dibenzo[fg,ij]phenanthro[9,10,1,2,3-pqrst]pentaphene. J. Mater. Chem. 2000, 10, 879-886.

3) Kubozono, Y.; Mitamura, H.; Lee, X.; He, X.; Yamanari, Y.; Takahashi, Y.; Suzuki, Y.; Kaji, Y.; Eguchi, R.; Akaike, K.; Kambe, T.; Okamoto, H.; Fujiwara, A.; Kato, T.; Kosugi, T.; Aoki, H. Metal-intercalated aromatic hydrocarbons: a new class of carbon-based superconductors. Phys. Chem. Chem. Phys. 2011, 13, 16476-16493.

4) Cox, E. G. Crystal Structure of Benzene. Rev. Mod Phys. 1958, 30, 159.

5) Kumar, B.; Viboh, R. L.; Bonifacio, M. C.; Thompson, W. B.; Buttrick, J. C.; Westlake, B. C.; Kim, M. S.; Zoellner, R. W.; Varganov, S. A.; Mörschel, P.; Teteruk, J.; Schmidt, M. U.; King, B. T. Septulene: The Heptagonal Homologue of Kekulene. Angew. Chem. Int. Ed. 2012, $51,12795-12800$.

6) Mitsuhashi, R.; Suzuki, Y.; Yamanari, Y.; Mitamura, H.; Kambe, T.; Ikeda, N.; Okamoto, H.; Fujiwara, A.; Yamaji, M.; Kawasaki, N.; Maniwa, Y.; Kubozono, Y. Superconductivity in alkali-metal-doped picene. Nature 2010, 464, 76-79.

7) Wang, X. F.; Liu, R. H.; Gui, Z.; Xie, Y. L.; Yan, Y. J.; Ying, J. J.; Luo, X. G.; Chen, X. H. Superconductivity at $5 \mathrm{~K}$ in alkali-metal-doped phenanthrene. Nat. Commun. 2011, 2, 507. 